\title{
CLASSICAL STRINGS AND BORN-INFELD EQUATIONS
}

A. AINSAAR, M. KOIV. KLASSIKALISED STRINGID JA BORN-INFELDI VORRANDID

A. АИНСААР, М. КЫИВ. КЛАССИЧЕСКИЕ СТРУНЫ И УРАВНЕНИЯ БОРНА-ИНФЕЛЬДА

(Presented by H. Keres)

So far two different forms of the string action integral have been proposed [ $\left.{ }^{1}\right]$ :

$$
\begin{aligned}
& S_{N}=m \int \sqrt{\sigma^{2}} d u^{0} d u^{1}, \\
& S_{S}=\int \sigma^{2} d u^{0} d u^{1}
\end{aligned}
$$

where

$$
\begin{gathered}
\sigma^{2}=-\frac{1}{2} \sigma^{\mu v} \sigma_{\mu \nu}, \\
\sigma^{\mu v}=\partial\left(x^{\mu}, x^{v}\right) / \partial\left(u^{0}, u^{1}\right) .
\end{gathered}
$$

Action (1), called the Nambu action, is a generalization of a free mass point, thus having a strict geometrical meaning. It leads to the following Lagrange-Euler equations:

$$
\partial / \partial u^{a}\left(\partial \sqrt{\sigma^{2}} \partial x_{, a}\right)=0
$$

where

$$
x_{, a}^{\mu}=\partial x^{\mu} / \partial u^{a} .
$$

This string model cannot be consistently quantized in an arbitrary space-time dimension. The Schild action (2) is hoped to be free from such difficulties $\left[{ }^{2}\right]$. As was shown by Nambu $\left[{ }^{3}\right]$, action (2) can be obtained by the Hamilton-Jacobi formalism basing on two-form relations. The equations of motion corresponding to (2) are

$$
\partial / \partial u^{a}\left(-\varepsilon^{a b} \sigma_{\mu v} x_{, b}^{\nu}\right)=0 .
$$

Action (1) is invariant under an arbitrary change of the parameters $u^{\prime}=u^{\prime}(u)$. This enables one to choose a part of the space co-ordinates as independent parameters, the rest being treated as fields [ $\left.{ }^{4}\right]$. As far as the choice is arbitrary, it leads to the equivalence of the co-ordinates and fields.

Let us take the case of two independent parameters of the string $u^{0}, u^{1}$ and an arbitrary number $m$ of space-time dimensions. Then, if one takes the space co-ordinates $x^{0}, x^{1}$ as new parameters with the transition Jacobian

$$
D=\partial\left(x^{0}, x^{1}\right) / \partial\left(u^{0}, u^{1}\right),
$$

then regardless of the action one can write 
where

$$
\begin{gathered}
\Phi=\Psi_{f g} \varepsilon^{f h} \Psi_{h i \varepsilon^{g i}}=\operatorname{det} \Psi, \\
\Psi_{f g}=\psi_{, f}^{\alpha} \psi_{, g}^{\alpha}+g_{f g}, \\
\psi_{, f}^{\alpha}=\partial \psi^{\alpha} / \partial x^{f} .
\end{gathered}
$$

Here Latin indices take the values 0 and 1 , the other co-ordinates, denoted by $\psi^{\alpha}(\alpha=1, \ldots, m-1)$, are considered as fields.

Equation (9) shows that the Nambu string is a sympleon $[4,5]$, i. e., the Lagrangian admits the factor $D$ at a reparametrization. The Lagrange-Euler equations of the Lagrangian in the new parameters

$$
L \sim \sqrt{\Phi},
$$

in accordance with the work by B. M. Barbashov and N. A. Chernikov $\left[{ }^{6}\right]$, prove to be the $(m-1)$-dimensional Born-Infeld equation system

$$
B^{\alpha}=0 \text {, }
$$

where

$$
B^{\alpha} \equiv \varepsilon_{i k} \varepsilon_{j l} \stackrel{\alpha}{\alpha}{ }_{i j} \Psi_{k l} .
$$

The Schild string is not a sympleon, for $D$ does not fall out of the action integral. Unlike the Nambu string case, the action integral cannot be written in such a way that independently of the parametrization, the variation of certain co-ordinates, called fields, would lead to field equations devoid of the initial parameters $u$. Still we pose the question whether the structure keeps the Born-Infeld equation in this case too.

Equations (7) split into two parts, where the first part corresponds to the values $\mu \rightarrow \alpha$ and the second one to $\mu=0,1$ :

$$
\begin{aligned}
& {\left[\varepsilon^{i f} \varepsilon^{k g}\left(\psi_{, i}^{\alpha} \Psi_{f g}\right)_{, k}-\varepsilon^{i f} \psi_{, i}^{\alpha} \Psi_{f g} U_{g}\right] D^{2}=0,} \\
& {\left[\varepsilon^{k g} \Psi_{f g, k}-\Psi_{f g} U_{g}\right] D^{2}=0,}
\end{aligned}
$$

where

$$
U_{g}=D^{-2} \varepsilon^{b a} \partial D / \partial u_{a} x_{, b}^{g} .
$$

Using the immediate consequence of (7)

$$
\partial \sigma^{2} / \partial u^{a}=0,
$$

one can give (17) the parametrization-independent form

$$
U_{g}=\varepsilon^{k g} \Phi_{, k} / 2 \Phi .
$$

Thus, in (16) the $u$-dependence has remained only in the factors $D^{2}$. Apart from it the following field equations ensue:

$$
\begin{gathered}
\varepsilon^{k g} \varepsilon^{i f} \varepsilon^{j m} \varepsilon^{n p}\left(\begin{array}{c}
\alpha \cdot i \\
\psi
\end{array} \Psi_{f g} \Psi_{j n} \Psi_{m p}+\psi_{, i}^{\alpha} \Psi_{f g, k} \Psi_{j n} \Psi_{m p}-\psi_{, i}^{\alpha} \Psi_{f g} \Psi_{j n} \Psi_{m p, k}\right)=0, \\
\varepsilon^{k g} \varepsilon^{j m} \varepsilon^{n p}\left(\Psi_{f g, k} \Psi_{j n} \Psi_{m p}-\Psi_{f g} \Psi_{j n} \Psi_{m p, k}\right)=0 .
\end{gathered}
$$

The following analysis of these equations in a usual way would be 
quite cumbersome and not straightforward. We worked out a graphical method for such purposes. At that an index corresponds to a graph vertex, 2-dimensional $\delta$ symbol is a line and $\varepsilon$ is an arrow between two vertices. The summing up over a function index $\alpha$ is denoted by a dotted line. Let us denote our functions as

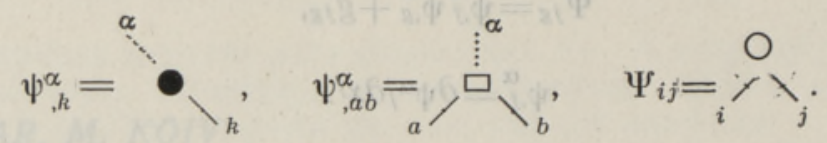

Then one can obtain immediately

$$
\Phi=\downarrow \downarrow \downarrow, \quad B \alpha=\downarrow \downarrow \downarrow
$$

It is an easy matter to find out general displacement rules for lines and arrows within a formula. All we need for our purpose is

$$
\begin{aligned}
& \underset{\rightarrow}{-}=\uparrow \mid+x, \\
& \rightarrow=\uparrow \uparrow+\uparrow,
\end{aligned}
$$

by which one can straightforwardly change equations (20) in the way that the Born-Infeld expression arises as a factor:

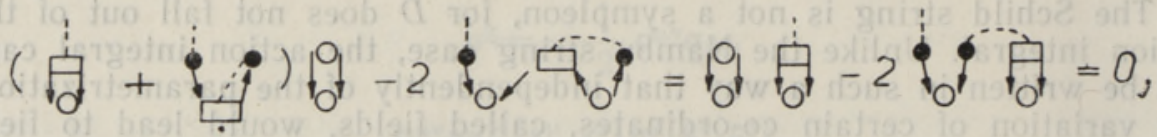

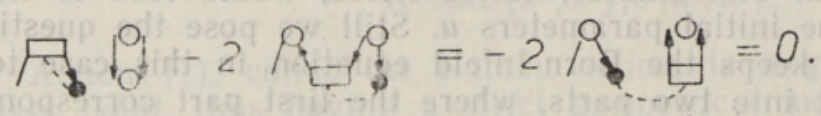

It can be rewritten at once:

$$
\begin{aligned}
& \left(2 \psi_{, i}^{\alpha} \varepsilon^{i f} \Psi_{f g} \varepsilon^{j d} \Psi_{, d}^{\beta}+\Phi \delta^{\alpha \beta}\right) B^{\beta}=0, \\
& 2 \Psi_{f j} \varepsilon^{j d} \psi,{ }_{d,}^{\beta} B^{\beta}=0,
\end{aligned}
$$

the nontrivial consequence of which is the corresponding Born-Infeld system of equations (14). In order to get a complete system corresponding to action (2) one can add to it equation (19).

\section{REFER E N C E S}

1. Schild, A., Phys. Rev., D16, № 6, 1722-1726 (1977).

2. E g u c h i, T., Phys. Rev. Lett., 44, № 3, 126-129 (1980).

3. N a m b u, Y., Phys. Lett., B92, № 3/4, 327-333 (1980).

4. A i ns a a r, A., Ki iranen, K., Kõ iv, M., ENSV TA Toimet., Füüs. Matem., 27, № 4, 453-455 (1978).

5. K õ i v, M., A i n s a a r, A., K i irane n, K., Preprint F-15, Tartu, 1981.

6. Ба рб ашов Б. М., Чер н и ков Н. А., Ж. эксперим. и теор. физ., 50, вып. 5 , $1296-1308$ (1966).

Academy of Sciences of the Estonian SSR, Institute of Physics

Received July 6,1981 